Check for updates

Cite this: Mater. Adv., 2021, 2, 4303

Received 18th January 2021, Accepted 8th May 2021

DOI: $10.1039 / \mathrm{d} 1 \mathrm{ma} 00042 j$

rsc.li/materials-advances

\section{Nontoxic photoluminescent tin oxide nanoparticles for cell imaging: deep eutectic solvent mediated synthesis, tuning and mechanism $\dagger$}

\author{
Laboni Das, (DD ${ }^{\text {ab }}$ Linmariya Devassy Koonathan, ${ }^{c}$ Amit Kunwar, (D) ab Suman Neogy, ${ }^{d}$ \\ Anil K. Debnath ${ }^{e}$ and Soumyakanti Adhikari (iD *a
}

\begin{abstract}
Non-toxic and photoluminescent (PL) tin oxide nanoparticle synthesis in Deep Eutectic Solvents (DESs) is being reported herein. Both radiation (electron beam and $\gamma$ radiation) and solvothermal methods were employed for the synthesis. An electron beam radiation technique proved to be more appropriate in tuning the size and morphology compared to the solvothermal process. Addition of any external oxidoreductive or stabilizing agent could be avoided by the use of Reline (choline chloride: urea; 1:2) as the host matrix. Detailed analysis of the PL behaviour of the nanoparticles is another important aspect of this study. The oxygen vacancies and tin interstitials responsible for photoluminescence have been identified from the de-convoluted PL spectra of the nanoparticles. Time dependent PL kinetics depicts PL decay at $\sim 1.2$ ns due to near band edge emission and at $\sim 3.15 \mathrm{~ns}$ due to defect state emission. The synthetic process has been standardized focusing on the size of the particles by varying all possible experimental parameters such as the temperature, concentration of the precursors, reaction time, dose of irradiation and dose rate. Synthesized nanoparticles have been characterized using XRD, XPS and EDX. TEM images illustrate nanomorphological differences obtained in the two methods. The probable mechanism of synthesis (both radiation and thermal) has been proposed based on the results obtained from transient studies using electron pulses and FTIR experiments. Cytotoxicity data demonstrate that the nanoparticles are suitable for application in biological studies involving cells up to a concentration of $10 \mu \mathrm{M}$. Imaging experiments with these photoluminescent nanoparticles exhibit their ubiquitous distribution including the nucleus of the tumour cells, which signifies potential application of these NPs for targeted drug delivery in cancer chemotherapy. Furthermore, the nanoparticles exhibited excellent antioxidant properties in vitro. The findings herein can open up enormous possibilities for more advanced and dedicated research towards using this cheap and versatile nanomaterial in a variety of biomedical applications.
\end{abstract}

\section{Introduction}

Deep Eutectic Solvents (DESs) are a newly emerging class of solvents consisting of hydrogen bonded structures of halide salts and hydrogen bond donors, and having a considerably lower melting point compared to their constituent components. ${ }^{1}$

\footnotetext{
${ }^{a}$ Radiation \& Photochemistry Division, Bhabha Atomic Research Centre, Mumbai 400 085, India. E-mail: asoumya@barc.gov.in; Fax: +91-22-25505331; Tel: $+91-22-25590301$

${ }^{b}$ HomiBhabha National Institute, Training School Complex, Anushaktinagar, Mumbai-400094, India

${ }^{c}$ Christ College (Deemed to be University), Bangalore, India

${ }^{d}$ Materials Science Division, Bhabha Atomic Research Centre, Mumbai 400085 , India

${ }^{e}$ Technical Physics Division, Bhabha Atomic Research Centre, Mumbai 400 085, India

† Electronic supplementary information (ESI) available. See DOI: 10.1039/d1ma00042j
}

They are appropriately being referred to as versatile alternatives to Room Temperature Ionic Liquids (RTILs) for their prominent advantages over the latter. Practically DESs possess all the remarkable properties of RTILs like negligible vapor pressure, tunable physical properties, nonflammability, wide electrochemical window etc., but excel over RTILs due to some added significant features like their easy and simple waste-free synthesis procedure, wherein no further purification is necessary. Furthermore, the initial raw materials for DES synthesis are much less expensive thereby making their large-scale production viable and cost effective. Also they are considered to be nontoxic and biodegradable compared to RTILs by nature. ${ }^{2}$ All these aforementioned features follow the principles of green chemistry and so preferably DESs are appropriate solvents for green synthesis of nanomaterials. Their low surface tension $(\sim 52 \mathrm{mN}$ in reline) leads to a high rate of nucleation and makes generation of small particles feasible (since Ostwald ripening is very weak). ${ }^{2}$ DESs are 
able to solubilize a large number of organic and inorganic compounds, which helps in overcoming the difficulty of working with several water insoluble precursors used in nanoparticle synthesis such as tin chloride used in the present study. However, compared to RTILs these are less explored in the field of nanosynthesis. Utilizing DESs as solvents for the synthesis of semiconductor nanomaterials has huge scope and possibilities.

Tin oxide is an important category of wide band gap (3.6 eV) n-type semiconductor material with proven potential for applications ${ }^{3}$ such as in electronics, ${ }^{4,5}$ optoelectronics, ${ }^{6}$ photovoltaics and dye sensitized solar cells, ${ }^{7}$ photocatalysis, ${ }^{8}$ rechargeable lithium ion batteries, ${ }^{9-11}$ gas sensors, ${ }^{12,13}$ and electrocatalysis. ${ }^{14}$ Due to its immense significance in nanotechnology, a large number of research reports have already appeared and innumerable methods are available in the literature for its synthesis. ${ }^{15-19}$ Among the metal oxide nanoparticles (MONPs) those of cadmium, arsenic, lead and mercury are observed to be toxic, whereas $\mathrm{TiO}_{2}, \mathrm{ZnO}$, $\mathrm{CuO}, \mathrm{Fe}_{3} \mathrm{O}_{4}$, and $\mathrm{Fe}_{2} \mathrm{O}_{3}$ are considered to be relatively safer for bioapplications. ${ }^{20} \mathrm{SnO}_{2}, \mathrm{In}_{2} \mathrm{O}_{3}$ and $\mathrm{Al}_{2} \mathrm{O}_{3}$ are less studied but they are expected to have low toxicity. ${ }^{21}$ Biogenically synthesized tin oxide NPs have shown significantly less cytotoxic and genotoxic effects. $^{22,23}$ Design of green methodologies for the synthesis of MONPs has a significant effect on lowering the toxicity so that they can be considered for bio-applications. ${ }^{24}$ However, tin oxide NPs are less explored in the area related to bio-applications.

Keeping in view the principles of green chemistry, herein a facile low-cost green synthesis method has been strategized by which we were successfully able to produce highly photoluminescent and biocompatible tin oxide nanoparticles.

Defect-related photoluminescence as observed in the present case is known to enhance photocatalytic, electro-catalytic and antibacterial effects due to trapping of electrons and holes in defect states. $^{25}$ However; here we have utilized the photoluminescence properties of the tin oxide NPs for a different purpose. To the best of our knowledge, photoluminescent tin oxide NPs have rarely been probed for cell imaging applications to date. Yan et al. have reported a multifunctional $\mathrm{SnO}_{2}$ nanoprobe for targeted cell delivery, and imaging and detection of microRNA. ${ }^{26}$ Of course, several metal oxide nanoparticles have been employed as biomedical agents in diagnostic, therapeutic and sensing platforms. ${ }^{27}$ For instance, nanosized $\mathrm{TiO}_{2}$ has shown its application in imaging; ${ }^{28}$ superparamagnetic iron oxide and manganese oxide have been used for molecular imaging. ${ }^{29}$ Nevertheless, photoluminescent bare tin oxide nanoparticles have not been employed for bio-imaging purposes, even though tin oxide has been seen to be less toxic than most other metal oxides. From cytotoxicity experiments, herein, we have established that the synthesized NPs are tolerated or less toxic. Accordingly, a cell imaging study has been carried out in both normal and cancer cells, wherein the obtained results are quite encouraging. This significant breakthrough for tin oxide NPs can open enormous possibilities for more advanced and dedicated research towards their use as biomedical materials.

A low-cost biodegradable solvent, Reline (DES), has been used as a green host matrix which itself acts as a stabilizing agent as well as a source of oxidizing radicals during radiation assisted synthesis. The possibilities, drawbacks and mechanistic details of both the thermo-chemical and radiation induced techniques in reline as a solvent have been studied in detail and a comparative analysis is presented herein.

\section{Experimental section}

\subsection{Chemicals}

High purity chemicals choline chloride $-\mathrm{ChCl}(\geq 98 \%)$, urea $\mathrm{NH}_{2} \mathrm{CONH}_{2}$ (99.5\%), tin(II)chloride dihydrate - $\mathrm{SnCl}_{2} \cdot 2 \mathrm{H}_{2} \mathrm{O}$ $(\geq 98 \%)$ and $2,2^{\prime}$-azinobis(3-ethylbenzothiazoline-6-sulfonic acid)diammonium salt (ABTS) were obtained from Sigma Aldrich and used as received. The organic solvents, absolute ethanol and acetone, were purchased from SD Fine-Chem Ltd, Mumbai, with the highest purity available. Nanopure water (conductivity, $0.06 \mu \mathrm{S} \mathrm{cm}^{-1}$ ) wherever used was obtained from the Millipore water purifying system. The water content in the DES was measured by the Karl-Fischer titration method (using a Metrohm $831 \mathrm{KF}$ Coulometer) and found to be $\sim 1000 \mathrm{ppm}$.

\subsection{Synthesis}

Prior to synthesis of reline, choline chloride and urea were separately vacuum dried at $90{ }^{\circ} \mathrm{C}$ and $60{ }^{\circ} \mathrm{C}$ respectively, for 48 hours. Reline was prepared by mixing $\mathrm{ChCl}$ and urea in a molar ratio of $1: 2$ followed by continuous heating and stirring at $80{ }^{\circ} \mathrm{C}$ (water bath) until a homogeneous colorless liquid was formed. The hydrogen bonded molecular structure of reline consisting of $\mathrm{ChCl}$ and urea in 1:2 molar ratio is shown in Fig. 1.

For the synthesis of tin oxide nanoparticles, $\mathrm{SnCl}_{2} \cdot 2 \mathrm{H}_{2} \mathrm{O}$ in measured quantity $(\sim 10 \mathrm{mM})$ was added to the previously prepared solvent, reline. In the thermo-chemical method, the reaction medium (along with the dissolved precursor) was subjected to continuous heating and stirring (700 rpm) for 90 minutes in a pre-heated water bath at $50{ }^{\circ} \mathrm{C}, 80^{\circ} \mathrm{C}$ and $98{ }^{\circ} \mathrm{C}$, respectively, for three different reaction set ups. White cloudiness started to appear after 15 minutes of reaction indicating the formation of nanoparticles; however, the reaction was further left for 90 minutes to attain completion. At $98{ }^{\circ} \mathrm{C}$, the appearance of a white cloudy precipitate was highest, and it was lowest at $50{ }^{\circ} \mathrm{C}$.

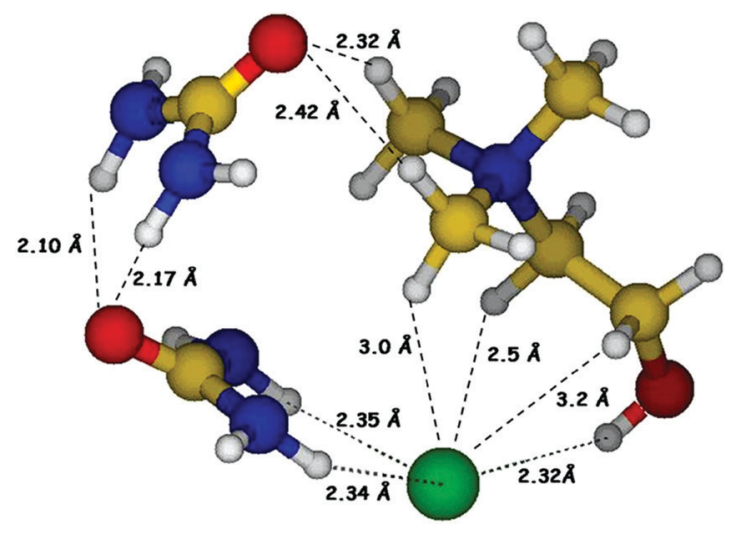

Fig. 1 Hydrogen bonded molecular structure of the deep eutectic solvent, reline. 
Experiments have been carried out by increasing the reaction time up to 3 hours and obtaining the absorbance at different time intervals which shows a decrease in absorption intensity of the nanoparticles after 2 hours (Fig. S1, ESI $\dagger$ ); henceforth the reaction time was optimized to 90 minutes. The concentration of the added precursor was increased from $10 \mathrm{mM}$ to $40 \mathrm{mM}$; however, no noticeable change in absorbance was observed. Colloidal suspension of the nanoparticles was precipitated using absolute ethanol. Ultrasonication and centrifugation of the solution yielded white precipitates. The precipitates were washed with ethanol 2-3 times and vacuum dried at $230{ }^{\circ} \mathrm{C}$ for $4-5$ hours followed by calcination at $400{ }^{\circ} \mathrm{C}$ and $800{ }^{\circ} \mathrm{C}$ for $2-5$ hours for further characterization.

For synthesis by the electron beam irradiation method, the precursor in measured quantity $(\sim 20 \mathrm{mM})$ was dissolved in reline by magnetic stirring only $(600 \mathrm{rpm})$ for approximately 2 hours (no heating was done). It has to be noted that although $\mathrm{SnCl}_{2} \cdot 2 \mathrm{H}_{2} \mathrm{O}$ forms a white precipitate of basic salt $\mathrm{Sn}(\mathrm{OH}) \mathrm{Cl}$ in water, it is solubilized to a clear solution in reline by stirring only. Furthermore, the clear solutions were irradiated by an electron beam with specific doses. However, no cloudiness or change in the color of the samples was observed after irradiation, which indicates that the nanoparticles formed are much smaller in size compared to those synthesized by a thermo-chemical route. On addition of ethanol to the irradiated medium, a white precipitate appeared which was centrifuged and calcined for further characterization as has been done in the case of the thermally synthesized particles.

\subsection{Antioxidant studies by ABTS radical scavenging assays}

ABTS free radical scavenging activity for the as synthesized NPS was experimentally determined according to the method reported in the literature. ${ }^{30}$ Stock solution was prepared by mixing ABTS $(7 \mathrm{mM})$ and potassium persulfate $(2.5 \mathrm{mM})$ and incubated at room temperature in the dark for 12-16 hours, to form $\mathrm{ABTS}^{\bullet+}$. The working solution was prepared by dilution of the stock solution by nanopure water (labelled as Solution A) such that it has an absorbance in the range of $0.70-0.80$ at $734 \mathrm{~nm}$. The NP stock solution prepared had a concentration of $12.74 \mathrm{mg} \mathrm{ml}^{-1}$ (6.37 $\mathrm{mg}$ of NPs dispersed in $0.5 \mathrm{ml}$ nanopure water). Different volumes (microliter) of the stock solution of NPs were added to $2 \mathrm{ml}$ of solution A and observed for 1 hour. With time, the bluegreen color of solution A bleached. After 1 hour the solutions were centrifuged to remove the un-dissolved NPs and the absorbance was observed at $734 \mathrm{~nm}$. The results obtained from UV-Vis absorption studies were plotted to determine the IC50 values of the NPs (i.e., concentration of the sample required to scavenge/ inhibit $50 \%$ of radicals). For comparison of antioxidant activity of the NPs with a standard, the IC50 value of ascorbic acid (a standard antioxidant) was also determined by following the same procedure and it was found to be $\sim 7.5 \mu \mathrm{g} \mathrm{ml}{ }^{-1}$.

\subsection{Cytotoxicity analysis by MTT assays}

Cytotoxicity studies were carried out in Chinese hamster ovary (CHO) cells, suspended in Dulbecco's modified Eagle's medium (DMEM) with $10 \%$ fetal calf serum seeded in a 96 well plate at a density of $3 \times 10^{3}$ cells per well and incubated at $37{ }^{\circ} \mathrm{C}$ in a $5 \%$ $\mathrm{CO}_{2}$ humidified atmosphere. After the attachment of cells (12-16 hours), they were treated with increasing concentrations of test compounds for 24 hours, and the cell viability was determined by a colorimetric MTT (3-(4,5-dimethylthiazol-2-yl)2,5-diphenyltetrazolium bromide) assay as described previously. ${ }^{31}$ The results have been presented as \% cytotoxicity calculated from the decrease in the characteristic absorbance of formazan crystals at $570 \mathrm{~nm}$ with respect to untreated cells i.e. control (100\%).

\subsection{Cell imaging}

The cellular imaging of nanoparticles was performed in normal Chinese Hamster Ovary (CHO) epithelial and human lung epithelial carcinoma (A549) cells maintained at Bhabha Atomic Research Centre. The cells were cultured in DMEM medium containing $10 \% \mathrm{FBS}, 100 \mathrm{U} \mathrm{ml}^{-1}$ penicillin and $100 \mu \mathrm{g} \mathrm{ml} \mathrm{m}^{-1}$ streptomycin and incubated at $37{ }^{\circ} \mathrm{C}$ in a humidified atmosphere with $5 \% \mathrm{CO}_{2}$. For imaging, $\sim 5 \times 10^{3}$ cells were grown on a cover slip, treated with $10 \mu \mathrm{M}$ concentration of nanoparticles for $24 \mathrm{~h}$ in cell culture medium, washed twice with $10 \mathrm{mM}$ phosphate buffered saline (PBS) and imaged using a fluorescence microscope (magnification, $10 \times$, Olympus) after excitation with an UV filter.

\subsection{Characterization}

Optical absorption measurements were carried out by using a JASCO V-650 absorption spectrophotometer. Photoluminescence studies have been carried out using a JASCO FP-8500 Spectrofluorometer. X-Ray diffraction (XRD) measurements were recorded on a Phillips X-ray diffractometer, model PW 1710 system, using a monochromatic $\mathrm{Cu} \mathrm{K}$ source $(\lambda=1.54 \AA$ ). The observed diffraction data were analyzed using the Fullprof-2000 Rietveld refinement program $^{32}$ in which the peak profile was fitted with the pseudoVoigt profile function and $\mathrm{U}, \mathrm{V}$, and $\mathrm{W}$ parameters were refined. In the initial stage the background parameters were adjusted with sixth order polynomial and an appropriate scale factor. The highresolution transmission electron microscopy (HRTEM) images were acquired on a Libra 200FE instrument. Samples for TEM measurements were prepared by re-dispersing the extracted powdered samples in ethanol and depositing a drop of this solution on a thin carbon coated copper grid followed by drying under an infrared lamp. The compositional analysis for the samples was performed with an energy dispersive X-ray spectrometer (EDX). X-Ray photoelectron spectroscopy (XPS) measurements were carried out using $\mathrm{Mg}-\mathrm{K}_{\alpha}(1253.6 \mathrm{eV})$ source and DESA-150 electron analyzer (StaibInstruments, Germany). The bindingenergy scale was calibrated to an adventitious C-1s line of $284.8 \mathrm{eV}$. The analyzer was operated at $40 \mathrm{eV}$ pass energy. The pressure in the chamber during analysis was $\sim 7 \times 10^{-9}$ torr. The anode voltage of the X-ray source was $15.5 \mathrm{kV}$, the filament current was 2.25 A and the emission current was $15.5 \mathrm{~mA}$. PL lifetime measurements were carried out by using a time correlated single photon counting (TCSPC) instrument (model: IBH, UK). The instrument response function (IRF) of the setup was measured by collecting the scattered light from a $\mathrm{TiO}_{2}$ suspension in water. The samples were excited by a DELTA LED of an output wavelength of $280 \mathrm{~nm}$ with an IRF of approximately $150 \mathrm{ps}$. 
The electron beam irradiation performed employing $7 \mathrm{MeV}$ electrons has been described elsewhere. ${ }^{33}$ For a $2 \mu$ s pulse, the measured dose was 145 Gy. Dosimetry was carried out using an air-saturated aqueous solution containing $5 \times 10^{-2} \mathrm{M} \mathrm{KSCN}$ $\left(\mathrm{G} \varepsilon=2.59 \pm 0.05 \mathrm{~m}^{2} \mathrm{~J}^{-1}\right.$ at $\left.475 \mathrm{~nm}\right) .{ }^{34}$ The FTIR spectra were recorded using a diamond single reflectance ATR probe in an IR Affinity-1 FTIR spectrometer. KF titration was carried out in an 831 KF Coulometer.

\section{Results and discussion}

For the thermo-chemically synthesized nanoparticles the color of the resulting solution was transparent at $50{ }^{\circ} \mathrm{C}$ and it turned cloudy white at a reaction temperature of $80{ }^{\circ} \mathrm{C}$ and the white color intensified at $98{ }^{\circ} \mathrm{C}$. The enhancement of cloudiness at higher temperatures obviously is due to an increase in nanoparticle concentration and agglomeration within the medium. Fig. 2A illustrates the appearance of an excitonic peak with the formation of nanoparticles at different temperatures. Blank absorption was taken before and after heating reline, which does not show any peak in the $200-300 \mathrm{~nm}$ wavelength region (Fig. 2A, a \& b).

The onset and mean position of the excitonic peak obtained at different reaction temperatures were red shifted, from $225 \mathrm{~nm}$ at $50{ }^{\circ} \mathrm{C}$ to $238 \mathrm{~nm}$ at $80{ }^{\circ} \mathrm{C}$ and $257 \mathrm{~nm}$ at $98{ }^{\circ} \mathrm{C}$. A bathochromic shift of the excitonic peak is mainly attributed to the increase in particle size. ${ }^{35}$ Furthermore, with an increase in reaction temperature considerable broadening of the excitonic peak was noticed, which corresponds mainly to the increase in the polydispersity of the nanoparticles. ${ }^{35-37}$ The direct band gap energy values were determined from the Tauc plot (Fig. 2A, inset) of $(\alpha h v)^{n} v s . h v$ (eqn (1)). The value of $n$ for a direct band gap semiconductor is 2 . The symbol ' $\alpha$ ' represents the absorption coefficient multiplied with the concentration of the nanoparticles,
Table 1 Data showing direct band gap energies calculated from the Tauc plot for nanoparticles synthesized via the thermo-chemical method and electron beam irradiation

\begin{tabular}{lll}
\hline & & Direct band gap $(\mathrm{eV})$ \\
\hline Temperature & $50{ }^{\circ} \mathrm{C}$ & 3.9 \\
& $80{ }^{\circ} \mathrm{C}$ & 3.3 \\
& $98^{\circ} \mathrm{C}$ & 2.8 \\
& & \\
Radiation dose & $35 \mathrm{kGy}$ & 4.2 \\
& $72 \mathrm{kGy}$ & 4.2 \\
& $107 \mathrm{kGy}$ & 4.7 \\
& $143 \mathrm{kGy}$ & 4.6
\end{tabular}

which was obtained from the relation $(2.303 A / l)$, where ' $A$ ' is the absorbance and ' $l$ ' is the optical path length of the cell $(1 \mathrm{~cm})$. The term ' $h v$ ' represents the photon energy.

$$
(\alpha h \nu)^{n}=K\left(h \nu-E_{\mathrm{g}}\right)
$$

The estimated direct band gap energies have been tabulated (Table 1), where a large blue shift with respect to a bulk band gap value of $3.6 \mathrm{eV}$ (direct band gap for $\mathrm{SnO}_{2}$ ) clearly indicates that the as grown nanoparticles belong to the strong confinement regime. Comparison of band gap values of the nanoparticles from table1 below shows that there is decrease in band gap with the increase in synthesis temperature. ${ }^{38}$ A concomitant decrease in band gap values with an increase in reaction temperature indicates the formation of larger particles at higher reaction temperature. The inverse relation of the band gap and particle size is well described using the following eqn (2)

$$
E_{\mathrm{g}}^{\mathrm{eff}}=E_{\mathrm{g}}+\hbar^{2} \pi^{2} / 2 \mu R^{2}-1.8 \mathrm{e}^{2} / \varepsilon R
$$

where $R$ is the particle radius, $\mu$ is the effective reduced mass, $E_{\mathrm{g}}$ is the bulk band gap energy ( $3.6 \mathrm{eV}$ for $\left.\mathrm{SnO}_{2}\right)$, and $E_{\mathrm{g}}^{\text {eff }}$ is the band gap of the nanoparticle.
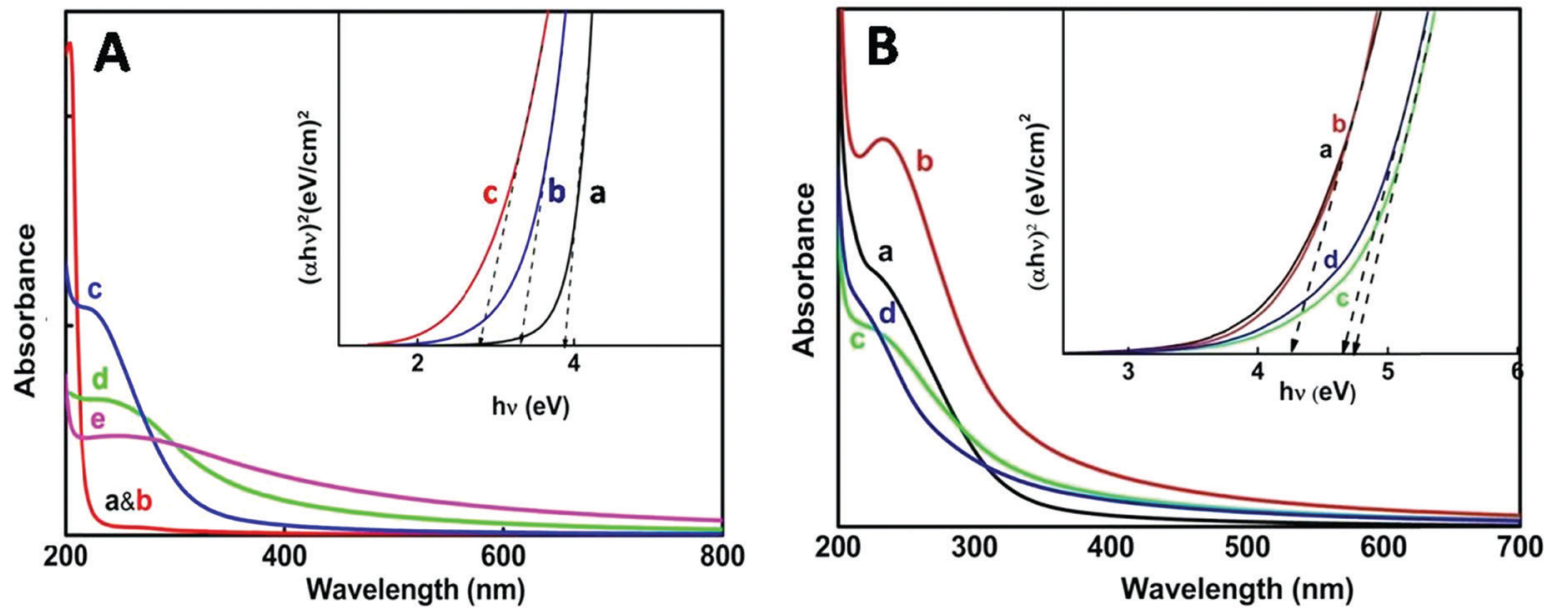

Fig. 2 (A) UV-Vis absorption spectra of tin oxide nanoparticles dissolved in nanopure water after separation from the host matrix, reline. (a) Only reline after heating at $80{ }^{\circ} \mathrm{C}$; (b) only reline without heating and (c) reline containing $\mathrm{SnCl}_{2} \cdot 2 \mathrm{H}_{2} \mathrm{O}$ heated at $50{ }^{\circ} \mathrm{C}$; (d) $80^{\circ} \mathrm{C}$; and (e) $98{ }^{\circ} \mathrm{C}$. Inset: direct band gap energy of the nanoparticles obtained from the Tauc plot. (a) $50^{\circ} \mathrm{C}$; (b) $80^{\circ} \mathrm{C}$; and (c) $98^{\circ} \mathrm{C}$. (B) UV-Vis absorption spectra of the as synthesized tin oxide nanoparticles dissolved in nanopure water after separation from the host matrix, reline, after e-beam irradiation at doses (a) 35 kGy, (b) 72 kGy, (c) 107 kGy (and d) 143 kGy. Inset: direct band gap energy of the nanoparticles obtained from the Tauc plot. (a) 35 kGy, (b) 72 kGy, (c) 107 kGy and (d) 143 kGy. 
Further experiments varying the reaction time of thermal synthesis and initial precursor concentration showed no change in the band gap of the NPs (Fig. S1, ESI $\dagger$ ).

In the case of nanoparticle synthesis via electron beam irradiation, the dose was varied from $35 \mathrm{kGy}$ to $143 \mathrm{kGy}$. The appearance of an excitonic peak after irradiation is shown in Fig. 2B. However, compared to the chemical synthesis peak broadening was not observed here, indicating uniform distribution of particle size. Even at a dose of $35 \mathrm{kGy}$ the particles produced were having a greater band gap $(\sim 4.2 \mathrm{eV})$ (Table 1$)$ than that obtained via thermo-chemical synthesis.

On increasing the dose above $100 \mathrm{kGy}$ particles of an even larger band gap were produced i.e., ultra-small particles could be obtained with the direct band gap as high as $\sim 4.6 \mathrm{eV}$. Ye et al. have reported ultrathin nanorods with a direct band gap of $\sim 4.72 \mathrm{eV} .{ }^{39}$ However, the excitonic peak obtained at $72 \mathrm{kGy}$ dose shows a better defined and sharper peak compared to the other doses which explains that the NPs obtained at this particular dose are confined to a very narrow size distribution range. Considering this we can conclude that $\sim 72 \mathrm{kGy}$ can be an optimum dose for the e-beam irradiation method under our experimental conditions. From the absorbance studies so far, we can infer that e-beam irradiation is a better method for the synthesis of ultra-small particles with minimum polydispersity.

X-Ray diffraction studies combined with Rietveld refinement were performed to define the crystal structure and chemical composition of the as synthesized nanoparticles. The extracted NPs in the powder form showed broad unresolved peaks (Fig. S2, ESI $\dagger$ ) which became more defined with an increase in calcination temperature up to $800{ }^{\circ} \mathrm{C}$ and the diffraction pattern of the nanoparticles was found to be in agreement with the standard JCPDS card no. 01-071-5323. ${ }^{40}$ No other XRD peak due to impurity phases including starting materials could be observed. Rietveld refined XRD data for compound $\mathrm{SnO}_{2}(\mathrm{~s})$ are shown in Fig. 3A. The derived cell parameters and residual parameters obtained from the refinement of XRD data for the compound is given in Table 2. Fig. 3B gives the crystal structure of $\mathrm{SnO}_{2}(\mathrm{~s})$ which was drawn using the VESTA program ${ }^{41}$ using
Table 2 Refined structural parameters for $\mathrm{SnO}_{2}(\mathrm{~s})$

\begin{tabular}{llll}
\hline Parameters & $\begin{array}{l}\text { Calculated values } \\
\text { after refinement }\end{array}$ & Parameters & $\begin{array}{l}\text { Calculated values } \\
\text { after refinement }\end{array}$ \\
\hline$a(\AA)$ & 4.73793 & $\mathrm{U}$ & 0.004123 \\
$b(\AA)$ & 4.73793 & $\mathrm{~V}$ & 0.007359 \\
$c(\AA)$ & 3.18709 & $\mathrm{~W}$ & 0.007132 \\
$\alpha\left({ }^{\circ}\right)$ & 90 & $\chi^{2}$ & 1.63 \\
$\beta\left({ }^{\circ}\right)$ & 90 & $R_{\text {exp }}$ & 11.23 \\
$\gamma\left({ }^{\circ}\right)$ & 90 & Space group & $P 42 / \mathrm{mnm}^{\circ}$ \\
$V\left(\AA^{3}\right)$ & 71.53260 & & \\
\hline
\end{tabular}

Rietveld refinement data. Calcination at higher temperatures $\left(400{ }^{\circ} \mathrm{C}\right.$ and $800{ }^{\circ} \mathrm{C}$ ) yields sharper peaks indicating the longrange ordering in the crystal. The prominent broad peaks obtained in XRD corresponding to (110), (101), (200) and (211) planes were considered for nanoparticle size determination.

Primarily the peak broadening in XRD depends upon two factors i.e., lattice strain and crystallite size, which are obtained from Williamson-Hall analysis. $4 \varepsilon \tan \theta$ gives the strain broadening and $K \lambda / D \cos \theta$ gives the broadening due to small crystallite size (Scherrer equation). Combining the two equations and rearranging gives eqn (3)

$$
\beta \cos \theta=K \lambda / D+4 \varepsilon \sin \theta
$$

$\beta \cos \theta$ was plotted against $4 \sin \theta$ wherein the slope and $y$-intercept of the fitted line represent the strain and size of the nanoparticle, respectively. The plot shows a negative strain of $-2.1 \%$ which probably is due to compressive strain or lattice shrinkage within the nanomaterial. From the intercept of the Williamson-Hall plot (Fig. S3, ESI $\dagger$ ) the particle size was calculated to be $\sim 8 \mathrm{~nm}$.

To confirm the stoichiometry of the synthesized compound and to get a clear view of the oxidation state of tin and oxygen, XPS analysis was carried out. Fig. 4 shows the XPS spectra of Sn-3d and O-1s peaks. The Sn-3d $\mathrm{d}_{5 / 2}$ peak (Fig. $4 \mathrm{~A}$ ) at $\sim 486.0 \mathrm{eV}$ and $\Delta E=8.4 \mathrm{eV}$ confirm the formation of $\mathrm{SnO}_{2}$. The $\mathrm{O}-1 \mathrm{~s}$ depicts a broad peak (Fig. 4B), which indicates the presence of an oxide peak $(\sim 530.0 \mathrm{eV})$, and adsorbed oxygen peaks at
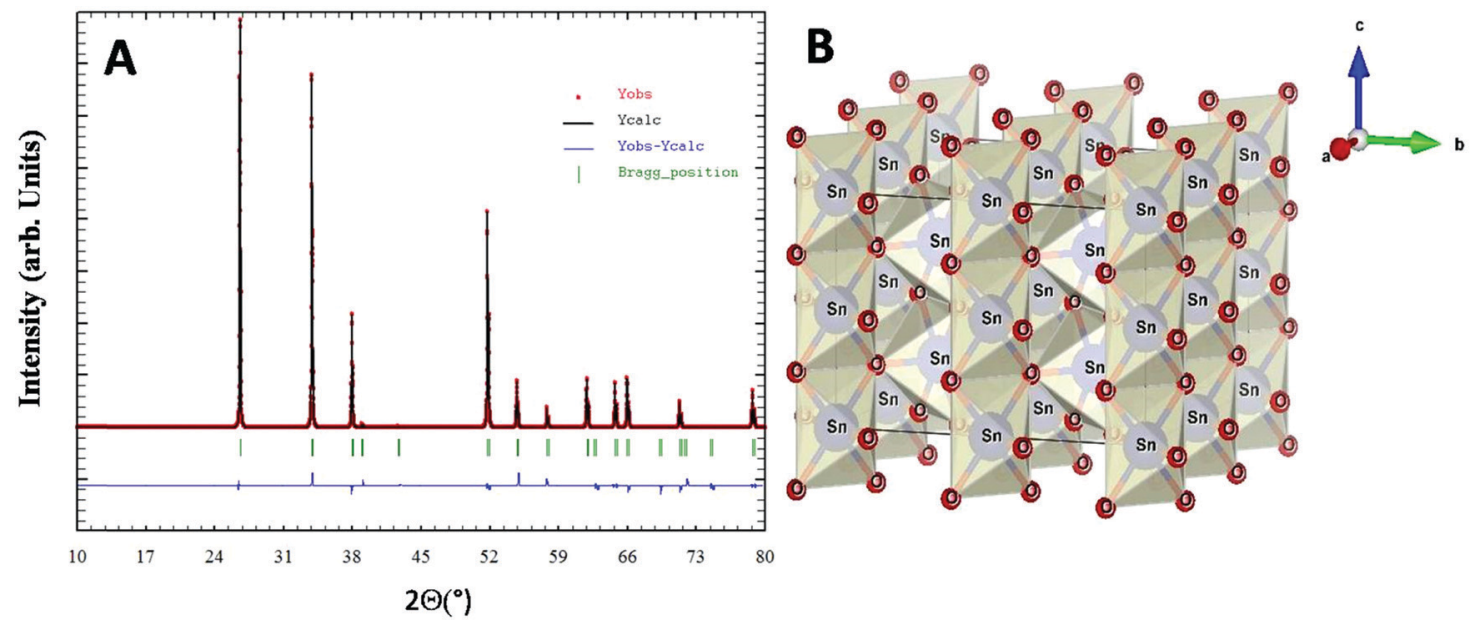

Fig. 3 (A) Rietveld refined XRD pattern of $\mathrm{SnO}_{2}(\mathrm{~s})$. (B) Crystal structure of $\mathrm{SnO}_{2}$. 

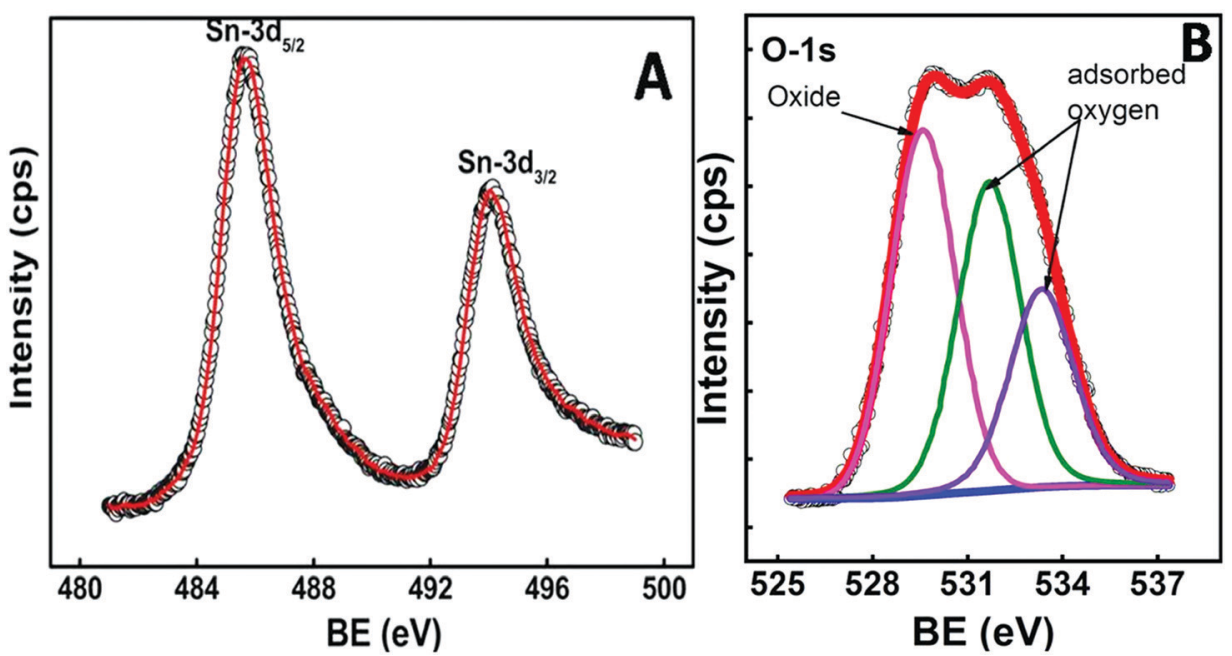

Fig. 4 Core level XPS spectra of (A) Sn-3d and (B) O-1s.

$531.6 \mathrm{eV}(-\mathrm{COOH})$ and $533.3 \mathrm{eV}(-\mathrm{C}-\mathrm{OH})$. Broadening of the $\mathrm{O}-1 \mathrm{~s}$ peak on the higher energy side indicates the presence of oxygen deficient tin oxide. ${ }^{42}$ From the XPS data, the $\mathrm{O} / \mathrm{Sn}$ ratio was calculated using eqn $(4)^{43}$

$$
C_{\mathrm{i}}=\frac{I_{\mathrm{i}} / S_{\mathrm{i}}}{\sum\left(\frac{I_{\mathrm{j}}}{S_{\mathrm{j}}}\right)}
$$

where $\mathrm{i}, \mathrm{j}=\mathrm{Sn}$ and $\mathrm{O}$. Here $I$ represents the intensity of the Sn-3d and O-1s peaks and is determined by finding the total area under the core level peak using the least-squares fitting of Gaussian line shape. In the case of the O-1s peak only the area of the oxide peak at $\sim 530.0 \mathrm{eV}$ was considered. $S$ is the atomic sensitivity factor and has values of 4.725 and 0.711 for $\mathrm{Sn}-3 \mathrm{~d}$ and $\mathrm{O}-1 \mathrm{~s}$ peaks, respectively. The $\mathrm{O} / \mathrm{Sn}$ ratio was found to be $\sim 1.986$, which is close to an ideal value of 2 . This result confirms the stoichiometry of the tin oxide nanoparticles.

The TEM images for the NPs obtained via e-beam irradiation depict self-assembling of the initially formed small spherical globules into larger sized ones, which further gets interconnected to form an entangled structure (Fig. 5). The interconnected structures formed were about micrometers in length (up to $1.5 \mu \mathrm{m}$ ). The globules obtained at doses up to $72 \mathrm{kGy}$ typically have a wide range of diameters (Fig. 5A and $\mathrm{B}$ ), with maximum particles within 80-90 nm. At higher dose ( $>100 \mathrm{kGy}$ ) the spheres were smaller in size with the diameter mostly within 55-65 nm (Fig. 5C and D). This was earlier indicated in absorbance studies where a higher band gap was obtained in NPs synthesized at dose $>100 \mathrm{kGy}$. Finally, it can be inferred that though the overall morphology is the same at all the four different e-beam doses, smaller particles could be obtained at doses $>100 \mathrm{kGy}$. Energy dispersive X-ray spectroscopy (EDS) carried out alongside the TEM imaging to confirm the composition of the synthesized nanomaterial (Fig. S4, ESI $\dagger$ ), depicting that the NPs are composed of Sn and O.
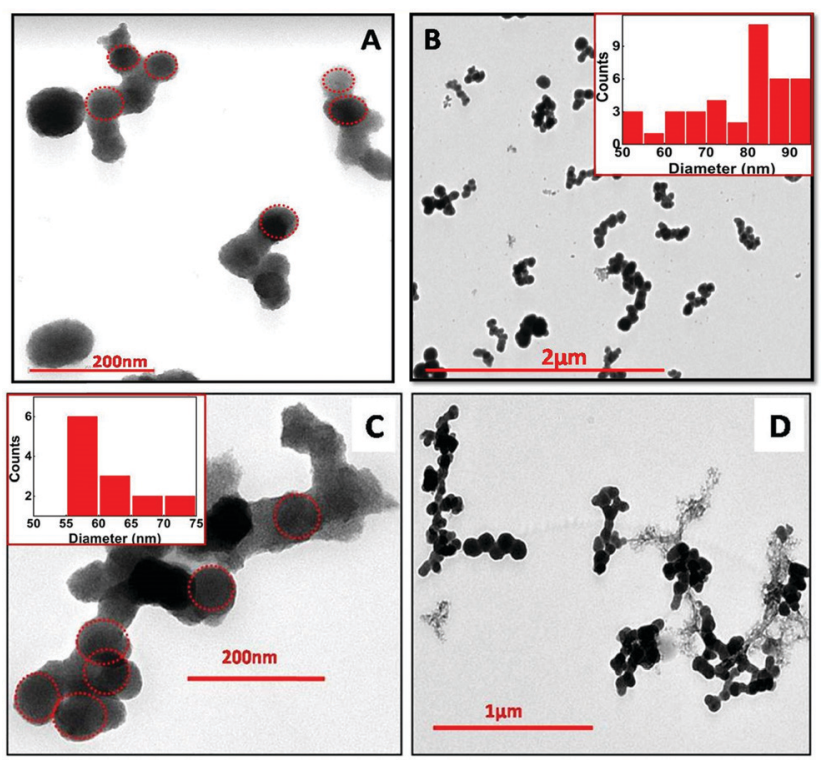

Fig. 5 TEM images of $\mathrm{SnO}_{2}$ NPs at different magnification, synthesized via electron beam irradiation. (A) 35 kGy, (B) 72 kGy, (C) 107 kGy and (D) 143 kGy in the reline matrix.

It is to be noted that although by electron beam radiation we were able to achieve a specific morphology, gamma radiation failed to provide definite structures (Fig. S5, ESI $\dagger$ ). It is pertinent to emphasize here that the dose rate involved in the electron beam irradiation technique was estimated to be $\sim 1.6 \mathrm{kGy} \mathrm{s}^{-1}$, while that in the case of $\gamma$-irradiation was $\sim 0.4 \mathrm{~Gy} \mathrm{~s}^{-1}$. Consequently, the high dose rate in the case of an electron beam leads to the instant deposition of a large amount of energy in a very short time. This aspect of the electron beam most likely is responsible for the difference in morphology compared to $\gamma$-irradiation as had been pointed out in an earlier report. ${ }^{44}$

The NPs synthesized by a solvothermal route exhibit elongated structures having a length of $\sim 50 \mathrm{~nm}$ (Fig. 6). The NPs synthesized at different reaction temperatures showed no difference in their 

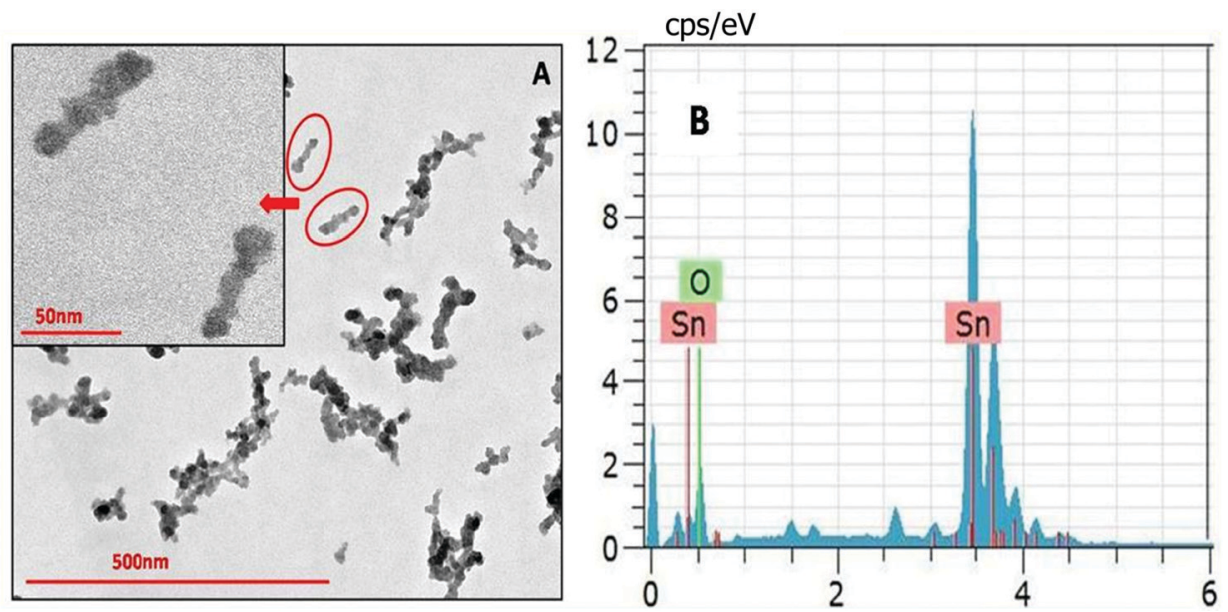

Fig. 6 (A) TEM image of $\mathrm{SnO}_{2}$ NPs obtained via a solvothermal route in the reline matrix. (B) The EDX spectrum of the $\mathrm{SnO}_{2}$ nanoparticles

morphology. Energy dispersive X-ray spectroscopy (EDS) has been carried out alongside the TEM imaging to confirm the composition of the synthesized nanomaterial. EDX data shown in Fig. 6B depict that the NPs are composed of Sn and O.
Fig. 7A and $\mathrm{C}$ shows the PL spectra of the $\mathrm{SnO}_{2}$ nanoparticles at $220 \mathrm{~nm}$ excitation wavelength. The emission spectra obtained at each individual temperature and at different e-beam radiation doses have been de-convoluted into five main peaks (Fig. 7B and D)
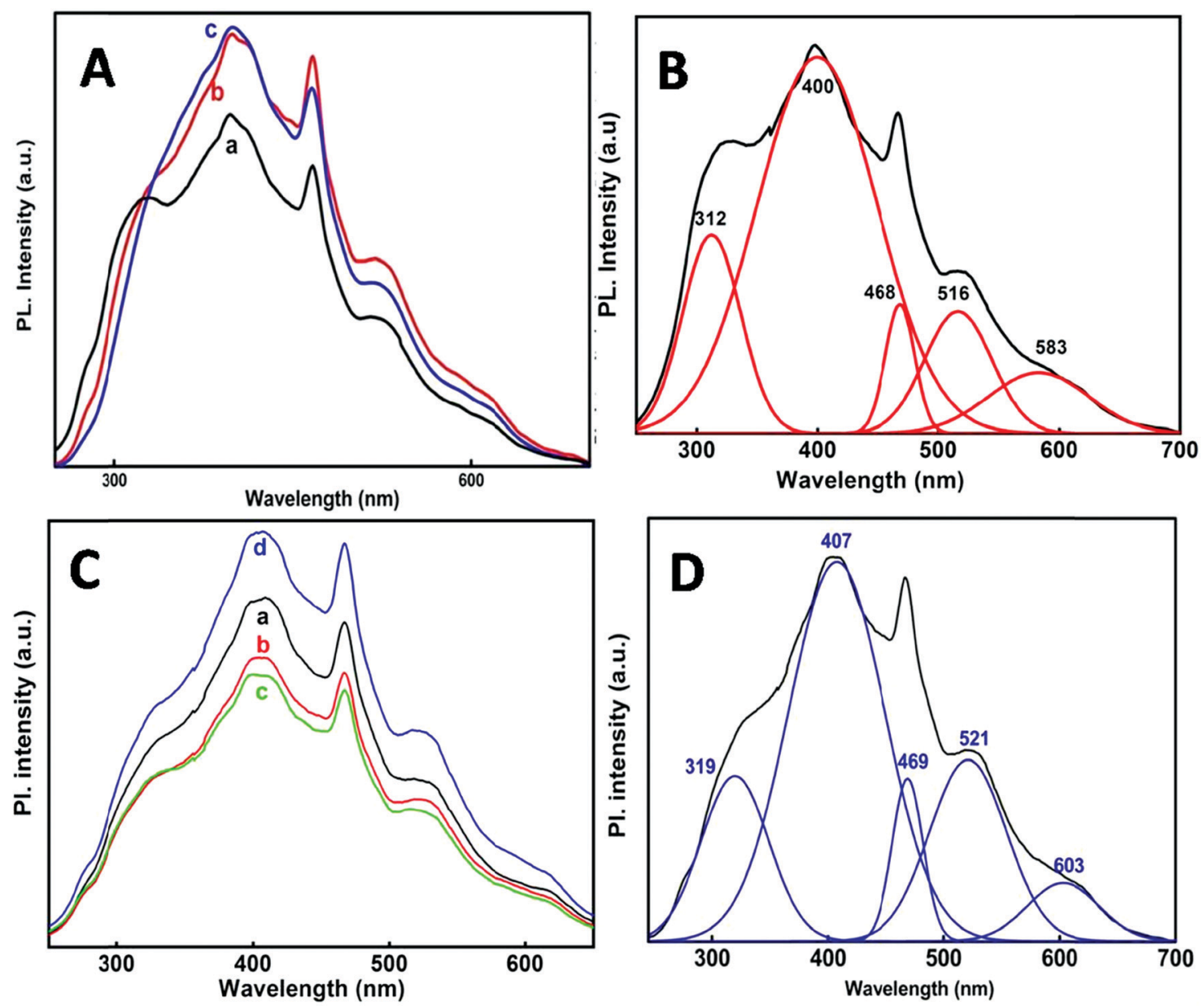

Fig. 7 (A) Room temperature PL spectra of tin oxide nanoparticles synthesized at different temperatures in reline $\left(\lambda_{\text {exc }}=220 \mathrm{~nm}\right)$, (a) $50{ }^{\circ} \mathrm{C}$; (b) $80{ }^{\circ} \mathrm{C}$; and (c) $98{ }^{\circ} \mathrm{C}$. (B) De-convoluted fitting plot of the PL spectrum of the nanoparticles obtained at $50{ }^{\circ} \mathrm{C}$ with $\lambda_{\text {exc }}=220 \mathrm{~nm}$. (C) Room temperature PL spectra of tin oxide nanoparticles synthesized at different e-beam irradiation doses in the reline host matrix ( $\lambda_{\text {exc }}=220 \mathrm{~nm}$ ), (a) $35 \mathrm{kGy}$; (b) $72 \mathrm{kGy}$; (c) 107 kGy and (d) 143 kGy. (D) De-convoluted fitting plot of the PL spectrum of the nanoparticles (by the e-beam irradiation method) at $\lambda_{\text {exc }}=220 \mathrm{~nm}$. 
Table 3 Peak maxima of the PL spectra obtained after de-convolution

\begin{tabular}{llllll}
\hline Synthesis & \multicolumn{5}{c}{ Peak position $\lambda(\mathrm{nm})$} \\
\hline $50{ }^{\circ} \mathrm{C}$ & 312 & 400 & 468 & 516 & 583 \\
$80{ }^{\circ} \mathrm{C}$ & 318 & 404 & 468 & 519 & 589 \\
$98{ }^{\circ} \mathrm{C}$ & 327 & 401 & 468 & 519 & 609 \\
via e-beam $(35,72,107,143 \mathrm{kGy})$ & 319 & 407 & 469 & 521 & 603
\end{tabular}

which are presented in Table 3. It is observed that one emission band is in the UV-region and the other four are in the visible region. Comparatively the emission in the visible region is much stronger than that in the UV region. A PL spectrum gives indication of the size and crystallinity of the $\mathrm{SnO}_{2}$ NPs. Earlier studies have shown that $\mathrm{SnO}_{2}$ NPs with larger size and perfectly crystalline structures show stronger UV emission. ${ }^{45-48}$ In the present case the NPs show stronger visible emission indicating that these are quite small in size and not perfectly crystalline as described from XRD.

The PL bands were found to be independent of excitation wavelength and found to have been originated from the "band gap" and "trapped state" or "defect level" emission. Upon excitation of the $\mathrm{SnO}_{2}$ NPs there is generation of an electronhole pair $\left(\mathrm{e}^{-}{ }_{\mathrm{CB}}\right.$ and $\left.\mathrm{h}_{\mathrm{VB}}^{+}\right)$. The first peak in the $\mathrm{UV}$ region ranging between 312 to $327 \mathrm{~nm}$ (considering different temperatures and different doses) can be ascribed to the near bandedge emission (NBE) of $\mathrm{SnO}_{2}$ (path 1, Scheme 1). The electrons first get excited to the highest level of the conduction band (CB); with time non-radiative relaxation (vibrational, without photon emission) occurs to the lowest energy level of the $\mathrm{CB}$ $\left(\mathrm{e}^{-}{ }_{\mathrm{CB}}\right)$ and then recombines with the holes in the valence band $\left(\mathrm{h}^{+}{ }_{\mathrm{VB}}\right)$ to give NBE (path 1 Scheme 1$)$. Patra et al. have observed a similar type of near band edge emission for $\mathrm{SnO}_{2}$ nanorods and nanoparticles. ${ }^{45}$ This type of emission which is not exactly "band gap emission" but has a lower energy than BG is known as "near band edge emission" and has already been reported by several other researchers. ${ }^{48-51}$ Mostly the visible light emission is known to be related to the defects arising from oxygen vacancies and $\mathrm{Sn}$ interstitials. The $\mathrm{SnO}_{2}$ host matrix as in the case of other metal oxides consists of oxygen vacancies which are created due to the escape of $\mathrm{O}^{2-}$ ions from the lattice. $\mathrm{V}_{0}^{\bullet}$

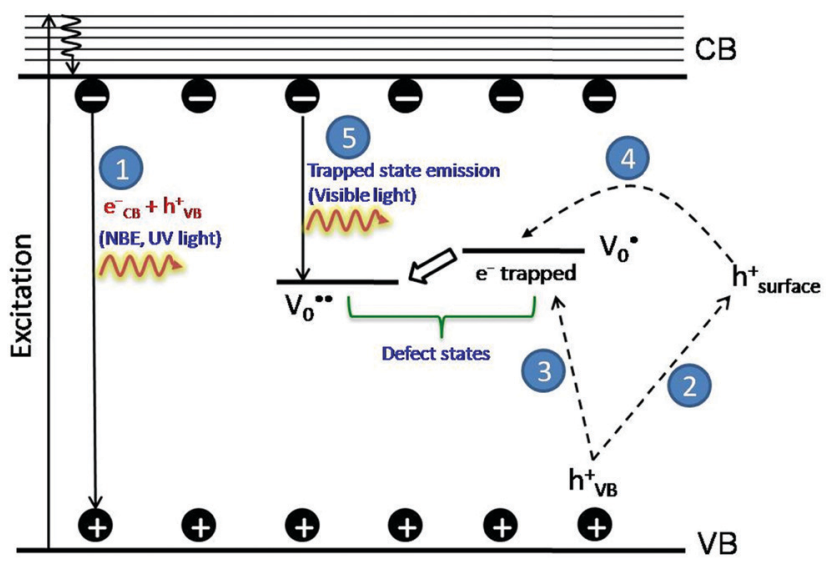

Scheme 1 Schematic diagram depicting the possible pathways of emission in tin oxide nanoparticles. states can be defined as electrons trapped within defects that are generated by the escape of oxygen atoms $\left(\mathrm{O}^{2-}\right)$; these are paramagnetic in nature having monopositive charge with respect to the $\mathrm{O}^{2-} \cdot \mathrm{h}^{+}$VB generated in the $\mathrm{VB}$ due to excitation can further progress through the reaction (5) (path 2, Scheme 1) trap at the surface of the particle and the (6) trap at the earlier generated $\mathrm{V}_{0}^{\bullet}$ centre to form a $\mathrm{V}_{0}^{\bullet \bullet}$ centre (path 3 , Scheme 1) ( 2 electron deficient w.r.t $\mathrm{O}^{2-}$ ). $\mathrm{h}^{+}$surface can further return back and recombine with an electron in $\mathrm{V}_{0}^{\bullet}$ to give $\mathrm{V}_{0}^{\bullet \bullet}$ (path 4 , Scheme 1)

$$
\begin{aligned}
& \mathrm{h}_{\mathrm{VB}}^{+} \rightarrow \mathrm{h}^{+} \text {surface } \\
& \mathrm{h}_{\mathrm{VB}}^{+}+\mathrm{V}_{0}^{\bullet} \rightarrow \mathrm{V}_{0}^{\bullet \bullet}
\end{aligned}
$$

$\mathrm{e}^{-}$Св can recombine with $\mathrm{V}_{0}^{\bullet \bullet}$ with emission in the visible region (path 5 , Scheme 1$).^{52}$ In the present study the emission in the visible region is due to such a recombination of conduction band electrons with a shallow and deep $\mathrm{h}^{+}$trap (i.e. $\mathrm{V}_{0}^{\bullet \bullet}$ ). These electrons which are trapped in the deep $\mathrm{e}^{-}$trap can return back to the $\mathrm{CB}$ and get captured by deep $\mathrm{h}^{+}$traps to give visible emission. ${ }^{50}$ This type of trapped state emission from shallow or deep traps is delayed emission and has longer lifetimes than the NBE. Time dependent PL studies have been carried out to explain it further and are discussed in the following section. The peaks above $500 \mathrm{~nm}$ are of low intensity and can be due to $\mathrm{O}$ vacancies or $\mathrm{Sn}$ interstitial defects. First principles calculations have already shown that the formation of $\mathrm{O}$ vacancies and Sn-interstitials is energetically favorable in the $\mathrm{SnO}_{2}$ lattice; so their existence in huge numbers is expected. ${ }^{51,53}$ All the visible region peaks are grossly denoted to the trapped states, $\mathrm{O}$ vacancies

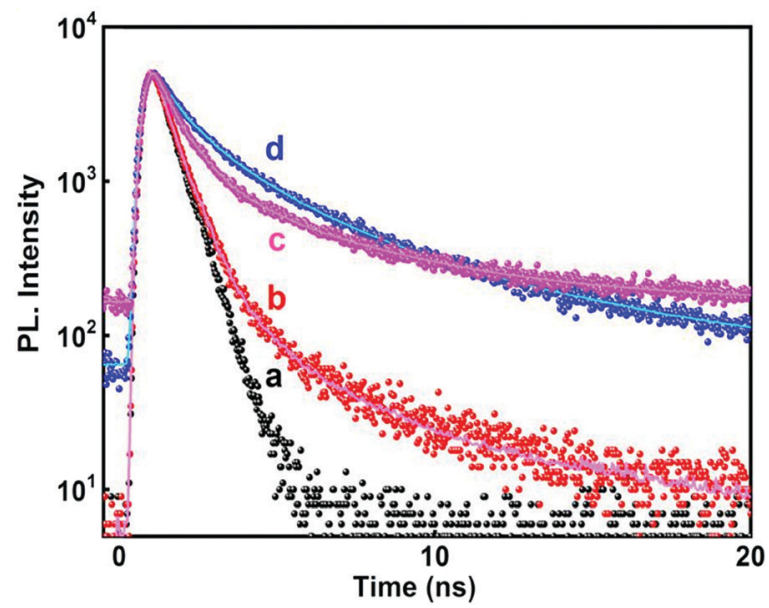

Fig. 8 Time resolved PL decay curve $\left(\lambda_{\text {exc }}=280 \mathrm{~nm}\right)$ of $\mathrm{SnO}_{2}$ nanoparticles at different emission wavelengths (a) IRF; (b) $320 \mathrm{~nm}$; (c) $467 \mathrm{~nm}$; and (d) $400 \mathrm{~nm}$.

Table $4 \mathrm{PL}$ lifetime values of $\mathrm{SnO}_{2}$ nanoparticles $\left(\lambda_{\mathrm{ex}}=220 \mathrm{~nm}\right)$ at three emission wavelengths $\left(\lambda_{\mathrm{em}}=320,400\right.$ and $\left.467 \mathrm{~nm}\right)$

\begin{tabular}{lllllllll}
\hline$\lambda(\mathrm{nm})$ & $T_{1}(\mathrm{~ns})$ & $B_{1}(\%)$ & $T_{2}(\mathrm{~ns})$ & $B_{2}(\%)$ & $T_{3}(\mathrm{~ns})$ & $B_{3}(\%)$ & $\chi^{2}$ & $\langle t\rangle(\mathrm{ns})$ \\
\hline 320 & 1.8 & 13 & 0.03 & 73 & 6.9 & 14 & 1.2 & 1.2 \\
400 & 2 & 28 & 6.6 & 36.3 & 0.74 & 35.6 & 1.1 & 3.2 \\
467 & 1.2 & 8.5 & 4.8 & 26 & 0.02 & 65 & 1.1 & 1.48
\end{tabular}


or Sn interstitials, but to identify the origin of each particular peak a more thorough and detailed investigation is required.

Noticeably the emission peaks (see Table 3) at three different reaction temperatures show a considerable red shift from

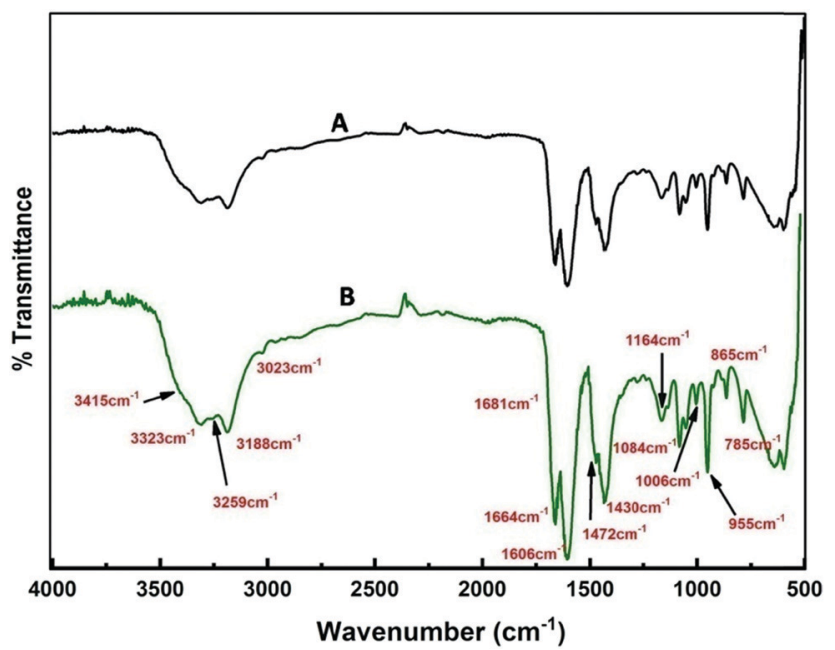

Fig. 9 FTIR spectra of (A) only reline and (B) reline containing dissolved $\mathrm{SnCl}_{2} \cdot 2 \mathrm{H}_{2} \mathrm{O}$ and heated at $80{ }^{\circ} \mathrm{C}$.
$312 \mathrm{~nm}$ to $327 \mathrm{~nm}$, which can be due to the subsequent decrease in the band gap with an increase in particle size with temperature as has been observed from UV-Vis spectra and the Tauc plot for the band gap (Fig. 2A and Table 1).

A time resolved study of PL decay has been carried out at all the emission wavelengths using a $280 \mathrm{~nm}$ excitation source. All the PL decay curves shown in Fig. 8 exhibited multiexponential behavior (tri-exponential) which were analyzed and fitted according to eqn (7)

$$
I(t)=a_{1} \mathrm{e}_{1}^{-t / \tau}+a_{2} \mathrm{e}_{2}^{-t / \tau}+a_{3} \mathrm{e}_{3}^{-t / \tau}
$$

where $I(t)$ is the time-dependent emission intensity, ' $a$ ' is the amplitude, and $\tau$ is the lifetime. The average PL lifetime $(\langle\tau\rangle)$ values were deduced from eqn (8)

$$
\langle\tau\rangle=\left(a_{1} \tau_{1}+a_{2} \tau_{2}+a_{3} \tau_{3}\right) /\left(a_{1}+a_{2}+a_{3}\right)
$$

The average lifetimes obtained after fitting and analyzing the decay traces have been presented in Table 4. For both the radiation assisted and thermally synthesized NPs, the NBE emission at $320 \mathrm{~nm}$ in the UV-region had fast decay kinetics. This fast PL decay had an average lifetime of $\sim 1.2 \mathrm{~ns}$ (although
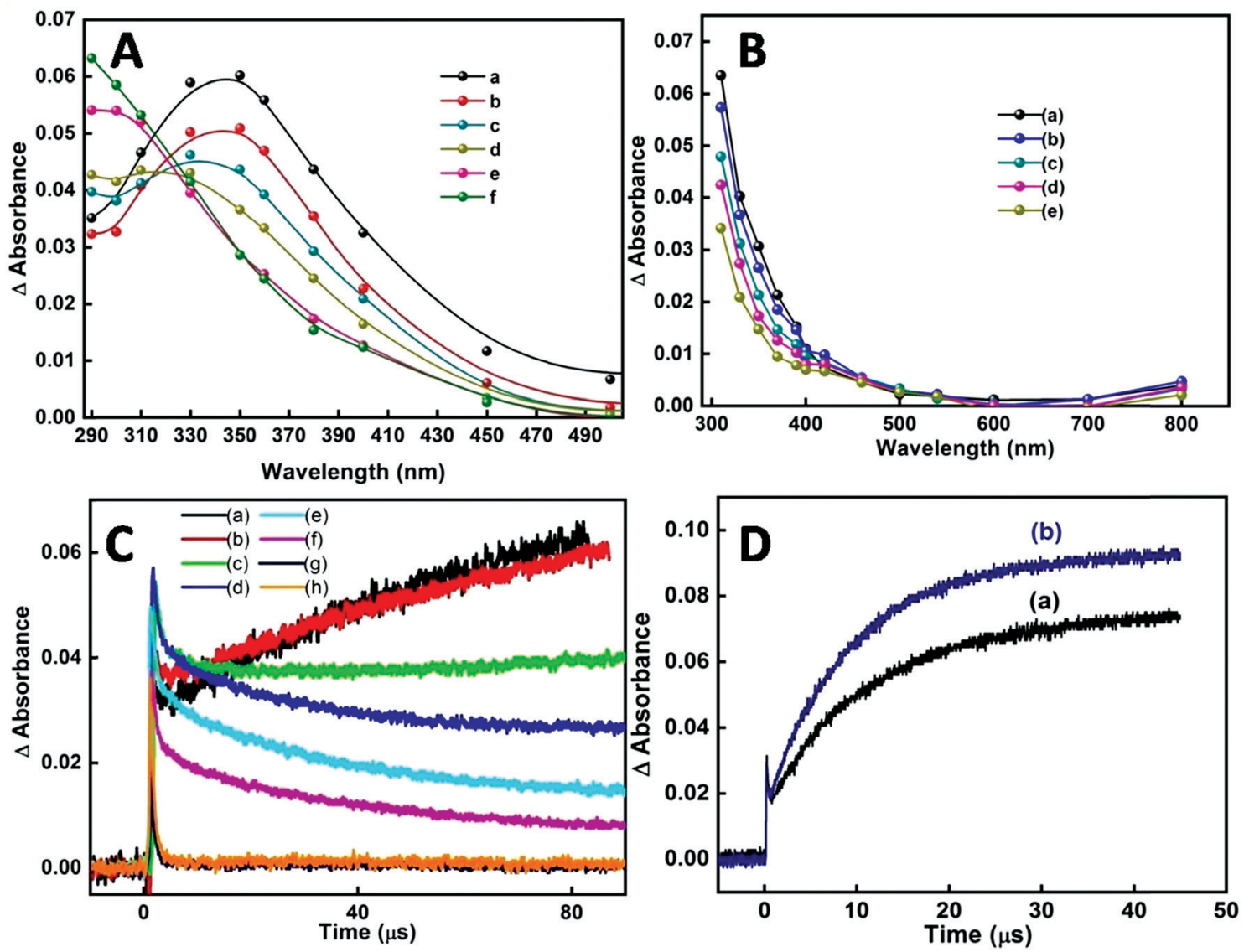

Fig. 10 (A) Transient absorption spectra of $\mathrm{N}_{2} \mathrm{O}$ purged reline containing $1 \mathrm{mM}$ tin chloride at (a) $0.8 \mu \mathrm{s}$, (b) $2.4 \mu \mathrm{s}$, (c) $11.2 \mu \mathrm{s}$, (d) $31.9 \mu \mathrm{s}$, (e) $78 \mu \mathrm{s}$ and (f) $89.2 \mu \mathrm{s}$ after the electron pulse. (B) Transient absorption spectra of $\mathrm{N}_{2} \mathrm{O}$ purged aqueous solution of $1 \mathrm{mM}$ tin chloride at (a) $2 \mu \mathrm{s}$, (b) $15.2 \mu \mathrm{s}$, (c) $31.2 \mu \mathrm{s}$, (d) $49.6 \mu \mathrm{s}$ and (e) $78.8 \mu \mathrm{s}$ after the electron pulse. (C) Kinetic traces of transient formation in $\mathrm{N}_{2} \mathrm{O}$ purged reline containing $1 \mathrm{mM} \mathrm{SnCl} \mathrm{l}_{2} \cdot 2 \mathrm{H}_{2} \mathrm{O}$ at (a) $300 \mathrm{~nm}$, (b) $310 \mathrm{~nm}$, (c) $330 \mathrm{~nm}$, (d) $350 \mathrm{~nm}$, (e) $370 \mathrm{~nm}$, (f) $390 \mathrm{~nm}$, (g) $500 \mathrm{~nm}$ and (h) $700 \mathrm{~nm}$. (D) Formation trace of ABTS ${ }^{\bullet+}$ at $414 \mathrm{~nm}$ in reline saturated with (a) argon and (b) $\mathrm{N}_{2} \mathrm{O}$. 


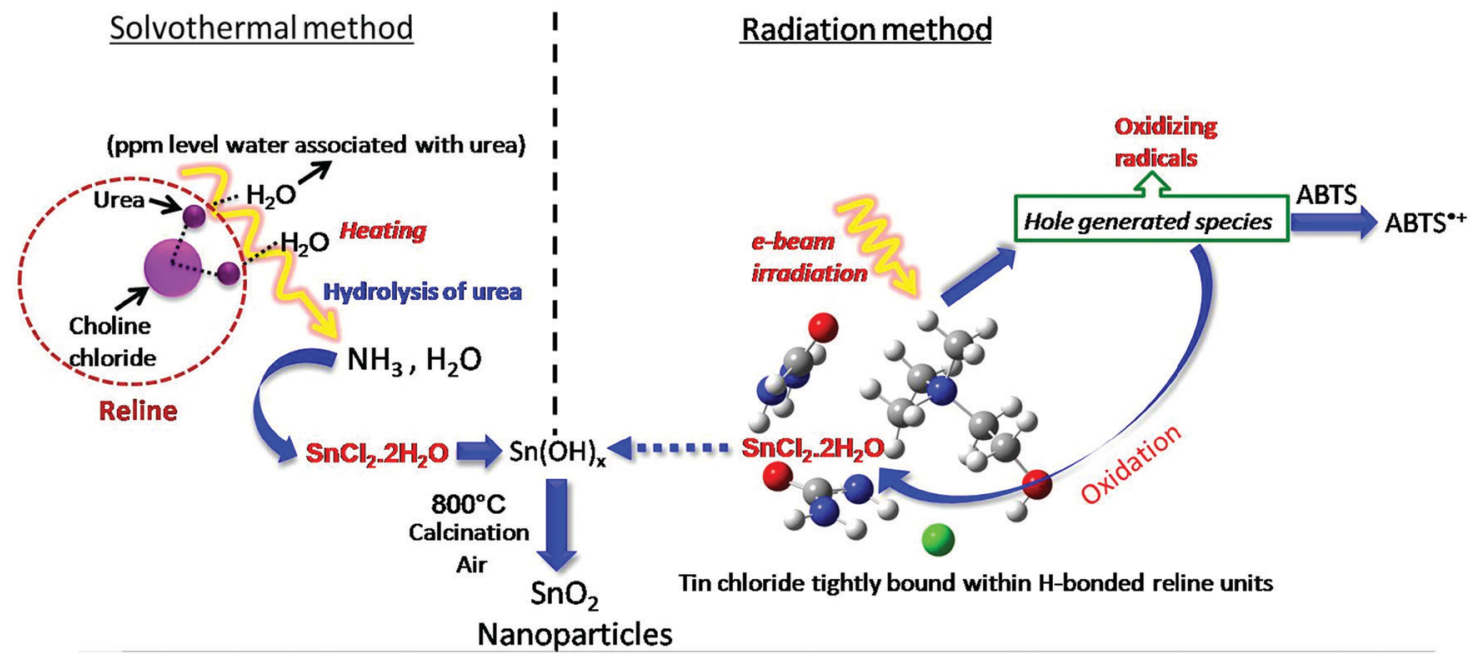

Scheme 2 Possible mechanism of formation of tin oxide nanoparticles by solvothermal and radiation methods.

it consisted of major contribution from the source itself, see Table 4) and is attributed to the NBE.

For the $400 \mathrm{~nm}$ emission, the decay is comparatively slower than the NBE emission and the average lifetime was found to be $\sim 3.15 \mathrm{~ns}$ indicating that these emissions are generated from defect states. Mostly the electrons recombine with the hole, de-trapping from the defect states as has already been discussed earlier. This is in agreement with most of the earlier reports which have mentioned decay times of binary semiconductor nanomaterials containing band gap and trapped state emission. ${ }^{46,47,54}$

A detailed analysis of the mechanism of NP synthesis carried out both for the solvothermal method and e-beam assisted method is described as follows. The water content of reline after preparation was measured to be $\sim 1000 \mathrm{ppm}$ by KF titration. The presence of water in the ppm level probably catalyzes the synthesis. Earlier reports suggest that the disruption of the reline structure only occurs above $41 \%$ of water addition..$^{55}$ To confirm the presence of water molecules and their role in nanoparticle synthesis in the present case, FTIR analysis was carried out. FTIR spectra of neat reline (with $\sim 1000 \mathrm{ppm}$ moisture) and thermally treated reline containing $\mathrm{SnCl}_{2} \cdot 2 \mathrm{H}_{2} \mathrm{O}$ is shown in Fig. 9. The water molecules present within reline remain mostly associated with urea. ${ }^{56}$ And in the present study this is inferred due to the following two reasons. Firstly, the bands at $3259 \mathrm{~cm}^{-1}, 1681 \mathrm{~cm}^{-1}$ and $1606 \mathrm{~cm}^{-1}$ observed in the present sample are characteristically found in urea-water mixtures ${ }^{56,57}$ and indicate the presence of water molecules near urea. However, the presence of vibrational bands at $785 \mathrm{~cm}^{-1}(\omega \mathrm{C}=\mathrm{O}), 865 \mathrm{~cm}^{-1}\left(\nu_{\mathrm{s}} \mathrm{N}-\mathrm{CH}_{3}\right)$, $955 \mathrm{~cm}^{-1}\left(\nu_{\text {as }} \mathrm{CCO}\right), 1006 \mathrm{~cm}^{-1}(\nu \mathrm{C}-\mathrm{O}), 1084 \mathrm{~cm}^{-1}\left(\rho \mathrm{CH}_{2}\right)$, $1164 \mathrm{~cm}^{-1}\left(\nu_{\text {as }} \mathrm{CN}\right)$, and $3023 \mathrm{~cm}^{-1}\left(\delta_{\mathrm{as}} \mathrm{OH}\right)$ indicates the formation of a eutectic between choline chloride and urea molecules. Secondly, shifting of vibrational bands occurs from a higher wave number (in reline without moisture) to lower wave numbers (in experimental samples) such as from $1669 \mathrm{~cm}^{-1}$ to $1664 \mathrm{~cm}^{-1}\left(\delta_{\mathrm{s}} \mathrm{NH}_{2}\right), 1446 \mathrm{~cm}^{-1}$ to $1430 \mathrm{~cm}^{-1}\left(\rho_{\mathrm{s}} \mathrm{NH}_{2}\right), 3349 \mathrm{~cm}^{-1}$ to $3323 \mathrm{~cm}^{-1}\left(\nu_{\mathrm{s}} \mathrm{NH}_{2}\right)$ and $3421 \mathrm{~cm}^{-1}$ to $3415 \mathrm{~cm}^{-1}\left(\nu_{\mathrm{as}} \mathrm{NH}_{2}\right)$.
All the earlier mentioned bands correspond to $\mathrm{NH}_{2}$ vibrations and their shift indicates the presence of hydrogen bonds between $\mathrm{H}_{2} \mathrm{O}$ molecules and $\mathrm{NH}_{2}$ which weakens $\mathrm{NH}_{2}$ bonds thus resulting in a red shift of vibrational bands. There is generation of ammonia on heating of the solvent and it remains mostly as ammonium hydroxide and catalyses the hydrolysis of $\mathrm{SnCl}_{2}$ to tin hydroxide. This is further transformed into tin oxide on calcination in air.

In our earlier transient absorption studies in argon purged neat reline,${ }^{58}$ formation of solvated electrons at $760 \mathrm{~nm}$ and holes or hole generated species at $340 \mathrm{~nm}$ was observed. In the present study both argon purged and $\mathrm{N}_{2} \mathrm{O}$ saturated reline containing dissolved $\mathrm{SnCl}_{2}$ has resulted in the formation of $\mathrm{SnO}_{2}$ nanoparticles. In both these solutions, pulse radiolysis studies indicate the appearance of a transient peak at around $290 \mathrm{~nm}$ (Fig. 10A). Also studies in $\mathrm{N}_{2} \mathrm{O}$ saturated aqueous solution of tin chloride have shown the appearance of a similar transient peak,

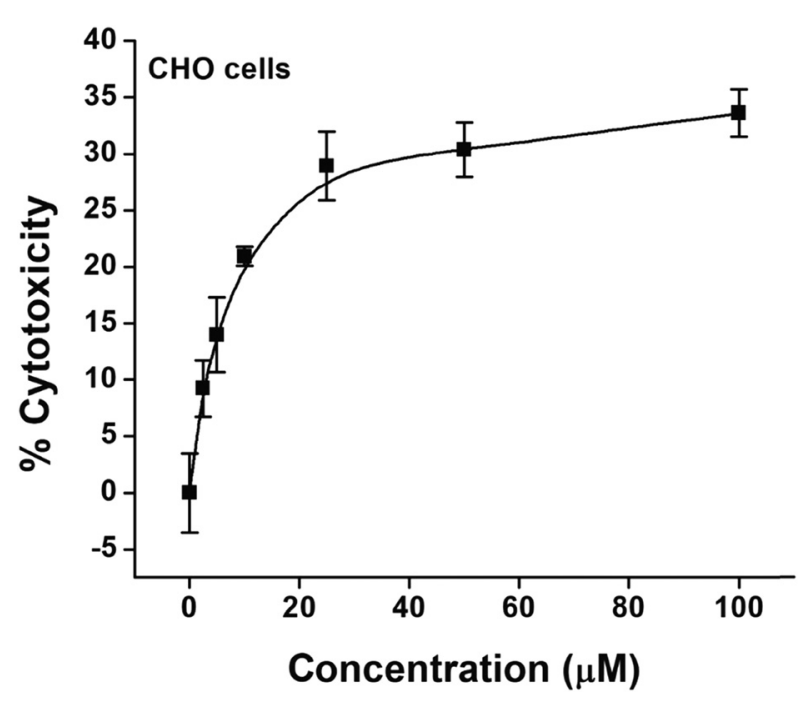

Fig. 11 Cytotoxic effects of increasing concentrations of the colloidal solution of $\mathrm{SnO}_{2}$ nanoparticles in $\mathrm{CHO}$ cells as determined by the MTT assay. Values are mean \pm SEM of three experiments. 
probably indicating the formation of the same transient species as that in the $\mathrm{SnCl}_{2}$-reline mixtures (Fig. 10B). At around $100 \mu$ s after the electron pulse, an increase in peak intensity at $290 \mathrm{~nm}$ with a subsequent decrease of the transient peak at $340 \mathrm{~nm}$ occurs in reline containing tin chloride. This is more evident from the decay traces and the formation traces of the new transient in Fig. 10C. This indicates that due to the interaction of reline with radiation initially the hole generated species is formed, which is absorbed at $340 \mathrm{~nm}$. These are responsible for producing the transient absorption at $290 \mathrm{~nm}$, which consequently evolves into the final compound. Furthermore, it was experimentally observed that the hole generated species oxidize in nature as they react with ABTS to form oxidized radical, the $\mathrm{ABTS}^{\bullet+}$ (Fig. 10D) radical.

Thus, essentially the mechanism of tin oxide nanoparticle generation by electron beam radiation in the reline host matrix
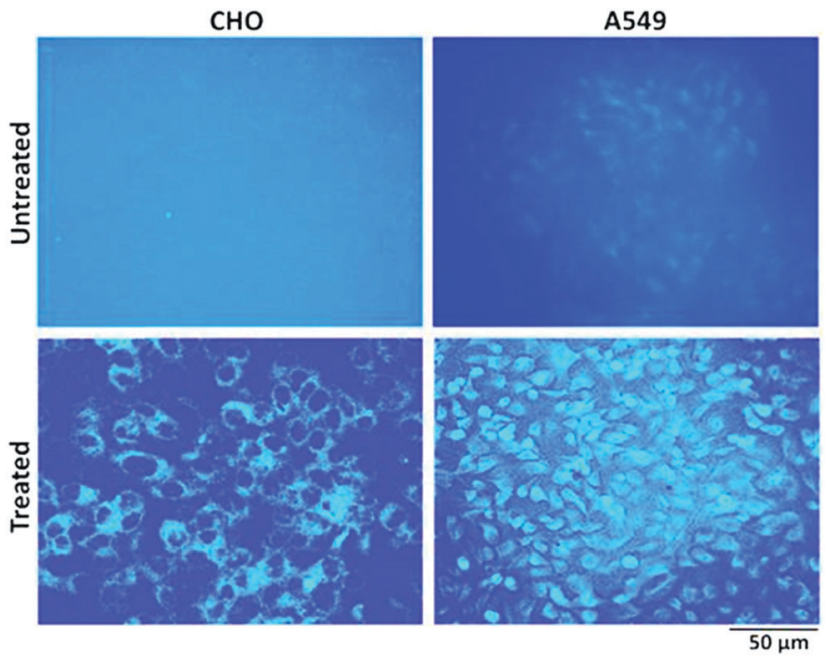

Fig. 12 Representative fluorescence images of $\mathrm{CHO}$ and $\mathrm{A} 549$ cells with and without NP $(10 \mu \mathrm{M})$ treatment for 24 hours. The images were acquired after excitation using an UV filter. Magnification $(10 \times)$. is through oxidation caused by the hole generated species. A possible mechanism of formation by both the methods is shown in Scheme 2.

The cytotoxic effect of NPs was evaluated in CHO cells. The results (Fig. 11) indicated a concentration dependent marginal increase in cytotoxicity up to $25 \mu \mathrm{M}$ while the cells were treated with NPs. A further increase in the concentration of NPs up to $100 \mu \mathrm{M}$ showed saturation with an overall cytotoxicity of merely $\sim 30 \%$. From this analysis, it was inferred that lower concentrations of NPs up to $10 \mu \mathrm{M}$ have negligible toxicity and are suitable for imaging studies.

To explore the possibility of a highly photoluminescent nature of the synthesized NPs in drug delivery and nanobiomedicines, ${ }^{27,59}$ cell imaging studies have been carried out. In comparison to untreated control cells, those treated with NPs showed bright blue fluorescence upon excitation using an UV filter (Fig. 12). Notably CHO cells showed only cytoplasmic localization of NPs, whereas A549 cells showed both cytoplasmic and nuclear localization of NPs. The reasons for the differential localization of NPs in tumour versus normal cells are not known. Nevertheless, the localization of NPs in the nucleus of tumour cells gains a lot of significance in view of its probable interaction with genomic DNA. DNA being the most critical target for anticancer drugs, it will be worth examining NPs for anticancer effects in future studies.

Several metal oxide NPs are found to possess intrinsic antioxidant activity due to their redox active nature. ${ }^{60}$ Herein free radical scavenging activity of the synthesized tin oxide NPs has been assayed using ABTS. The radical cation $\mathrm{ABTS}^{\bullet+}$ is blue green in color which gets bleached in the presence of the nanoparticles, indicating the antioxidant activity of NPs. ${ }^{61}$ The antioxidant properties are mainly either due to hydrogen atom transfer (HAT) or single electron transfer (SET). In the present case there are chances of single electron transfer due to the excess surface electrons or the electrons trapped in defect states present in the NPs. Fig. 13A shows an IC50 value of the NPs of $\sim 430 \mu \mathrm{g} \mathrm{ml} \mathrm{m}^{-1}$. For comparison with a standard
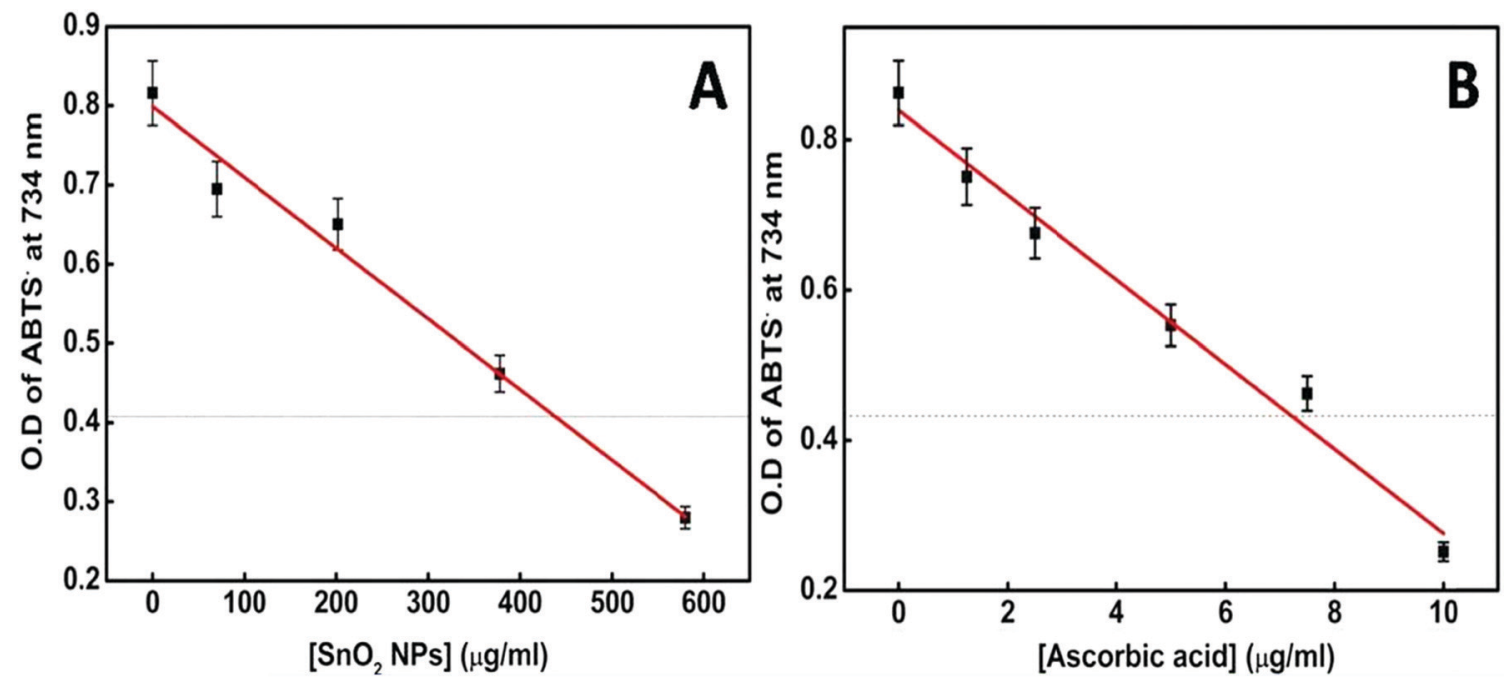

Fig. 13 (A) Plot of [ $\mathrm{SnO}_{2} \mathrm{NPs}$ ] vs. OD of the ABTS radical at $734 \mathrm{~nm}$. (B) Plot of [ascorbic acid] vs. OD of the ABTS radical at $734 \mathrm{~nm}$. 
antioxidant, the $\mathrm{ABTS}^{\bullet+}$ scavenging activity of pure ascorbic acid was estimated and its IC50 value was found to be $\sim 7.5 \mu \mathrm{g} \mathrm{ml}^{-1}$ (Fig. 13B) following the same procedure. Philip et al. have shown the antioxidant activity of $\mathrm{SnO}_{2}$ by scavenging free radicals of DPPH. ${ }^{62}$ Selenium nanoparticles (Se NPs) are known to be good antioxidants. Comparing the IC50 value of $\mathrm{SnO}_{2}$ NPs with that of amorphous Se NPs ${ }^{63}$ shows that the presently synthesized $\mathrm{SnO}_{2}$ performs better.

\section{Conclusions}

In summary, DESs can be considered as low-cost versatile green solvents, as well as stabilizing media for the synthesis of nanoparticles. Both e-beam and gamma radiation can be employed to synthesize photoluminescent tin oxide NPs in reline. However, the results have shown that with gamma irradiation no specific morphology is achievable, whereas in the case of e-beam the NPs can attain definite spherical structures. In the e-beam irradiation technique it is preferable to work within the dose range of 70-100 $\mathrm{kGy}$, where both the size and polydispersity of the NPs would be optimum. The solvothermal route also produces photoluminescent $\mathrm{SnO}_{2} \mathrm{NPs}$, however, the morphology consisted of elongated structures. The shape and size of the NPs are very important aspects in most of the biological applications where cellular uptake is necessary. ${ }^{64}$ Thus, depending on the field of application of the NPs one has to decide whether the e-beam irradiation or solvothermal method would be the method of preference.

The photoluminescent $\mathrm{SnO}_{2}$ NPs showed marginal cytotoxicity even up to a concentration of $100 \mu \mathrm{M}$, which rendered them safe for cell imaging studies, wherein exciting results could be obtained in tumor cells. Preferential interactions of the NPs with DNA (within nucleus) of cancer cells pave the way for exploring possibilities of their application in anticancer effects or imaging of deep tissues and tumor cells. Recently, nanoantioxidants have been gaining popularity due to their effective performance as therapeutic nanomedicines mainly in neuronal damage caused by oxidative stress, where conventional antioxidants are unable to cross the blood brain barrier. ${ }^{65}$ The results of antioxidant studies involving the as synthesized $\mathrm{SnO}_{2}$ NPs reveal excellent properties to inhibit oxidative damage by reactive radical species.

\section{Author contributions}

Laboni Das conceived the idea, designed and performed the experiments and wrote the manuscript. Linmariya Devassy Koonathan performed the experiments and helped in the literature survey. Amit Kunwar conducted the cytotoxicity and cell imaging experiments and analysed and wrote the results and discussion part of cytotoxicity and cell imaging. Suman Neogy carried out the TEM imaging and EDX analysis of the samples. Anil K. Debnath performed XPS experiments and wrote the XPS portion. Soumyakanti Adhikari supervised the overall project, provided critical feedback and helped with the analysis of the manuscript.

\section{Conflicts of interest}

There are no conflicts of interest to declare.

\section{Acknowledgements}

The authors are thankful to the members of the RPCD LINAC facility for their help in the irradiation work, and Mr Amit Kanjilal for the FTIR experiments. The authors thank $\mathrm{Mr}$ Sumanta Mukherjee for his help in XRD studies and Rietveld analysis. The authors thank Dr Awadhesh Kumar, Head, RPCD and Dr A. K. Tyagi, Associate Director, Chemistry Group, BARC, for their constant support and encouragement.

\section{References}

1 E. L. Smith, A. P. Abbott and K. S. Ryder, Chem. Rev., 2014, 114, 11060-11082.

2 D. V. Wagle, H. Zhao and G. A. Baker, Acc. Chem. Res., 2014, 47, 2299-2308.

3 D. Mohanta and M. Ahmaruzzaman, RSC Adv., 2016, 6, 110996-111015.

4 R. Medhi, C.-H. Li, S. H. Lee, M. D. Marquez, A. J. Jacobson, T.-C. Lee and T. R. Lee, ACS Appl. Nano Mater., 2019, 2, 6554-6564.

5 S. D. Ponja, B. A. D. Williamson, S. Sathasivam, D. O. Scanlon, I. P. Parkin and C. J. Carmalt, J. Mater. Chem. C, 2018, 6, 7257-7266.

6 K. C. Pradel, Y. Ding, W. Wu, Y. Bando, N. Fukata and Z. L. Wang, ACS Appl. Mater. Interfaces, 2016, 8, 4287-4291.

7 G. Shang, J. Wu, S. Tang, M. Huang, Z. Lan, Y. Li, J. Zhao and X. Zhang, J. Mater. Chem., 2012, 22, 25335-25339.

8 D. Zhao and X. Wu, Mater. Lett., 2018, 210, 354-357.

9 Z. Liu, Z. Cao, B. Deng, Y. Wang, J. Shao, P. Kumar, C. R. Liu, B. Wei and G. J. Cheng, Nanoscale, 2014, 6, 5853-5858.

10 S. Li, Y. Wang, C. Lai, J. Qiu, M. Ling, W. Martens, H. Zhao and S. Zhang, J. Mater. Chem. A, 2014, 2, 10211-10217.

11 S. Ding and X. Wen Lou, Nanoscale, 2011, 3, 3586-3588.

12 Y. Cao, Y. Li, D. Jia and J. Xie, RSC Adv., 2014, 4, 46179-46186.

13 R. Ghosh, A. K. Nayak, S. Santra, D. Pradhan and P. K. Guha, RSC Adv., 2015, 5, 50165-50173.

14 L. Jiang, G. Sun, Z. Zhou, S. Sun, Q. Wang, S. Yan, H. Li, J. Tian, J. Guo, B. Zhou and Q. Xin, J. Phys. Chem. B, 2005, 109, 8774-8778.

15 G. Cheng, J. Wang, X. Liu and K. Huang, J. Phys. Chem. B, 2006, 110, 16208-16211.

16 V. Juttukonda, R. L. Paddock, J. E. Raymond, D. Denomme, A. E. Richardson, L. E. Slusher and B. D. Fahlman, J. Am. Chem. Soc., 2006, 128, 420-421.

17 K. P. Gattu, K. Ghule, A. A. Kashale, V. B. Patil, D. M. Phase, R. S. Mane, S. H. Han, R. Sharma and A. V. Ghule, RSC Adv., 2015, 5, 72849-72856.

18 M. Lai, J. A. Gonzalez Martinez, M. Grätzel and D. J. Riley, J. Mater. Chem., 2006, 16, 2843-2845.

19 Y. Wang, J. Y. Lee and T. C. Deivaraj, J. Mater. Chem., 2004, 14, 362-365. 
20 R. Augustine, A. P. Mathew and A. Sosnik, Appl. Mater. Today, 2017, 7, 91-103.

21 A. M. C. Ng, M. Y. Guo, Y. H. Leung, C. M. N. Chan, S. W. Y. Wong, M. M. N. Yung, A. P. Y. Ma, A. B. Djurišić, F. C. C. Leung, K. M. Y. Leung, W. K. Chan and H. K. Lee, J. Photochem. Photobiol., B, 2015, 151, 17-24.

22 S. M. Roopan, S. H. S. Kumar, G. Madhumitha and K. Suthindhiran, Appl. Biochem. Biotechnol., 2015, 175, 1567-1575.

23 M. J. Khan and Q. Husain, Prep. Biochem. Biotechnol., 2014, 44, 558-571.

24 A. B. Seabra and N. Durán, Metals, 2015, 5, 934-975.

25 M. Zimbone, M. A. Buccheri, G. Cacciato, R. Sanz, G. Rappazzo, S. Boninelli, R. Reitano, L. Romano, V. Privitera and M. G. Grimaldi, Appl. Catal., B, 2015, 165, 487-494.

26 H. Dong, J. Lei, H. Ju, F. Zhi, H. Wang, W. Guo, Z. Zhu and F. Yan, Angew. Chem., Int. Ed., 2012, 51, 4607-4612.

27 M. P. Nikolova and M. S. Chavali, Biomimetics, 2020, 5, 27.

28 K. T. Thurn, E. Brown, A. Wu, S. Vogt, B. Lai, J. Maser, T. Paunesku and G. E. Woloschak, Nanoscale Res. Lett., 2007, 2, 430-441.

29 P. Padmanabhan, A. Kumar, S. Kumar, R. K. Chaudhary and B. Gulyás, Acta Biomater., 2016, 41, 1-16.

30 A. Guleria, S. Chakraborty, S. Neogy, D. K. Maurya and S. Adhikari, Chem. Commun., 2018, 54, 8753-8756.

31 T. Mosmann, J. Immunol. Methods, 1983, 65, 55-63.

32 Rodriguez-Carvajal, J. Fullprof 2000 Version 1.6, Laboratoire Leon Brillouin, Gifsur Yvette, France, 2000.

33 S. N. Guha, P. N. Moorthy, K. Kishore, D. B. Naik and K. N. Rao, Proceedings of the Indian Academy of Sciences Chemical Sciences, 1987, 99, 261-271.

34 G. V. Buxton and C. R. Stuart, J. Chem. Soc., Faraday Trans., 1995, 91, 279-281.

35 L. Das, A. Guleria, S. Neogy and S. Adhikari, Colloids Surf., A, 2018, 555, 781-786.

36 A. Guleria, S. Singh, M. C. Rath, A. K. Singh, S. Adhikari and S. K. Sarkar, J. Lumin., 2012, 132, 652-658.

37 A. K. Ganguli, A. Ganguly and S. Vaidya, Chem. Soc. Rev., 2010, 39, 474-485.

38 T. Yang, M. Zhu, K. Gu, C. Zhai, Q. Zhao, X. Yang and M. Zhang, New J. Chem., 2018, 42, 13612-13618.

39 G. Xi and J. Ye, Inorg. Chem., 2010, 49, 2302-2309.

40 G. Xiao, Y. Wang, J. Ning, Y. Wei, B. Liu, W. Yu, G. Zou and B. Zou, $R S C$ Adv., 2013, 3, 8104-8130.

41 K. Momma and F. Izumi, J. Appl. Crystallogr., 2011, 44, 1272-1276.

42 M. Periyasamy and A. Kar, J. Mater. Chem. C, 2020, 8, 4604-4635.
43 D. Schild, in Hydrogen Technology: Mobile and Portable Applications, ed. A. Léon, Springer Berlin Heidelberg, Berlin, Heidelberg, 2008, pp. 575-601, DOI: 10.1007/978-3-540-69925-5_23.

44 L. Das, A. Guleria, S. Neogy and S. Adhikari, RSC Adv., 2016, 6, 92934-92942.

45 A. Kar, S. Kundu and A. Patra, J. Phys. Chem. C, 2011, 115, 118-124.

46 M. Fang, L. Zhang, X. Tan, X. Hu, W. Yan and P. Liu, J. Phys. Chem. C, 2009, 113, 9676-9680.

47 M. A. Z. G. Sial, M. Iqbal, Z. Siddique, M. A. Nadeem, M. Ishaq and A. Iqbal, J. Mol. Struct., 2017, 1144, 355-359.

48 M. Fang, X. Tan, B. Cheng and L. Zhang, J. Mater. Chem., 2009, 19, 1320-1324.

49 Y. Dai, Y. Zhang, Q. K. Li and C. W. Nan, Chem. Phys. Lett., 2002, 358, 83-86.

50 H. Kaur, H. Bhatti and K. Singh, J. Mater. Sci.: Mater. Electron., 2019, 30, 2246-2264.

51 M. Bhatnagar, V. Kaushik, A. Kaushal, M. Singh and B. R. Mehta, AIP Adv., 2016, 6, 095321.

52 F. Gu, S. F. Wang, M. K. Lü, G. J. Zhou, D. Xu and D. R. Yuan, J. Phys. Chem. B, 2004, 108, 8119-8123.

53 J. X. Zhou, M. S. Zhang, J. M. Hong and Z. Yin, Solid State Commun., 2006, 138, 242-246.

54 L. Das, A. Guleria and S. Adhikari, $R S C A d v ., 2015,5$, 61390-61397.

55 P. Kumari, Shobhna, S. Kaur and H. K. Kashyap, ACS Omega, 2018, 3, 15246-15255.

56 C. Du, B. Zhao, X.-B. Chen, N. Birbilis and H. Yang, Sci. Rep., 2016, 6, 29225.

57 Y. Zhang, J. Han and C. Liao, J. Electrochem. Soc., 2016, 163, D689-D693.

58 L. Das and S. Adhikari, J. Phys. Chem. B, 2018, 122, 8900-8907. 59 J. K. Patra, G. Das, L. F. Fraceto, E. V. R. Campos, M. d. P. Rodriguez-Torres, L. S. Acosta-Torres, L. A. Diaz-Torres, R. Grillo, M. K. Swamy, S. Sharma, S. Habtemariam and H.-S. Shin, J. Nanobiotechnol., 2018, 16, 71.

60 L. Valgimigli, A. Baschieri and R. Amorati, J. Mater. Chem. B, 2018, 6, 2036-2051.

61 R. Re, N. Pellegrini, A. Proteggente, A. Pannala, M. Yang and C. Rice-Evans, Free Radical Biol. Med., 1999, 26, 1231-1237.

62 V. K. Vidhu and D. Philip, Spectrochim. Acta, Part A, 2015, 134, 372-379.

63 A. Guleria, D. K. Maurya, S. Neogy, B. S. Raorane, A. K. Debnath and S. Adhikari, New J. Chem., 2020, 44, 4578-4589.

64 S. Dasgupta, T. Auth and G. Gompper, Nano Lett., 2014, 14, 687-693.

65 R. Sandhir, A. Yadav, A. Sunkaria and N. Singhal, Neurochem. Int., 2015, 89, 209-226. 